\title{
Nível de conhecimento do Profissional de Educação Física frente a alunos com hipertensão arterial nas academias de ginástica
}

\author{
Level of knowledge of the Physical Education Professional \\ working with hypertensive students in gyms
}

\author{
Luciana Zaranza Monteiro \\ Itana Lisane Spinato \\ Carlos Antônio Bruno da Silva \\ Zélia Maria de Sousa Araújo Santos 2 \\ Renan Magalhães Montenegro Júnior
}

1 Universidade de São Paulo. Faculdade de Medicina de Ribeirão Preto. Departamento de Clínica Médica. Divisão de Endocrinologia e Metabologia. Ribeirão Preto, SP. Brasil.

\section{Universidade de} Fortaleza. Programa de Pós-graduação em Saúde Coletiva. Fortaleza. CE, Brasil.

3 Universidade Federal do Ceará. Departamento de Saúde Comunitária da Faculdade de Medicina. Fortaleza. CE, Brasil.

Recebido em 26/05/09 Revisado em 27/08/09 Aprovado em 12/10/09
Resumo - A hipertensão arterial é um dos principais fatores de risco para o desenvolvimento de doenças cardiovasculares e atinge parcela considerável da população brasileira. O objetivo deste estudo foi avaliar o nível de conhecimento dos Profissionais de Educação Física que atuavam com alunos hipertensos, comparando-os com os profissionais que não atuavam. Participaram 400 profissionais que atuavam nas academias de ginástica do Município de Fortaleza, CE. Foi utilizado um questionário sobre o conhecimento de critérios da hipertensão arterial, tipos de exames laboratoriais solicitados, tipo de hipertensão dos alunos, cuidados gerais para a prática de atividade física e recomendações para adquirir um melhor controle da hipertensão. Dos 400, 302 (75,5\%) atuavam com alunos hipertensos, destes, 236 (59\%) baseavam-se apenas na informação do aluno para o diagnóstico de hipertensão. A respeito das situações em que os profissionais contra-indicavam a atividade física para alunos hipertensos, 130 (43,1\%) informaram PA elevada, 47 (15,5\%) nenhuma situação e $(21,5 \%)$ não sabiam informar. Sobre a mensuração da PA antes e depois da atividade física, observamos que a maioria $(62,3 \%)$ dos profissionais que atuavam com alunos hipertensos não verificava a PA. O conhecimento desses profissionais acerca da hipertensão arterial em academias foi insuficiente. Sugere-se que estudos mais específicos sejam realizados a fim de obterem-se dados que auxiliem na elaboração de programas voltados à atuação do profissional que esclareçam as necessidades do aluno hipertenso nas academias de ginástica.

Palavras-chave: Conhecimento; Hipertensão Arterial; Educação em Saúde; Atividade Física.

Abstract - Arterial hypertension is one of the main risk factors for the development of cardiovascular diseases and affects a substantial part of the Brazilian population. The objective of this study was to compare the level of knowledge between Physical Education Professionals who worked with hypertensive students in gyms and those who did not. A total of 400 professionals working in gyms in the Municipality of Fortaleza-CE participated in the study. A questionnaire containing the following information was used: knowledge about criteria for hypertension, types of laboratory tests, types of hypertension in the students, general care during physical activity, and recommendations for a better control of hypertension. Among the 400 professionals, 302 (75.5\%) worked with hypertensive students. Of these, 236 (59\%) based their diagnosis of hypertension solely on the report of the students. Regarding the situations in which the professionals contraindicated physical activity for the hypertensive students, $43.1 \%$ reported high blood pressure, $15.5 \%$ reported no situation, and $21.5 \%$ had no information. With respect o blood pressure measurement before and after physical activity, most of the professionals (62.3\%) working with hypertensive students did not measure the student's blood pressure. The knowledge of the professionals about hypertension in gyms was insufficient. More specific studies are necessary to obtain data that will contribute to the development of programs designed for Physical Education Professionals that establish the needs of hypertensive students in gyms.

Key words: Knowledge; Hypertension; Health education; Physical education. 


\section{INTRODUÇÃO}

A hipertensão arterial (HA) é considerada um dos fatores de risco mais importante para o desenvolvimento de doenças cardiovasculares ${ }^{1}$, representando no Brasil um dos problemas de saúde pública de maior prevalência na população, capaz de levar a óbito, aproximadamente, $40 \%$ dos indivíduos acometidos ${ }^{2}$. O tratamento da HA é realizado por meio de medicamentos e deve estar associado a uma mudança de estilo de vida, como alterações no padrão alimentar e prática regular de exercícios físicos ${ }^{3}$.

Sua prevalência varia de acordo com cada país, podendo ser encontrado valores de $4 \%$ na China e mais de $30 \%$ nos EUA. No Brasil, as taxas variam de 22 a $43 \%$, dependendo da região do país ${ }^{4,5}$. Parte considerável dos pacientes hipertensos apresenta comorbidade com diabetes, obesidade e dislipidemia, além de outros fatores de risco, como sedentarismo e tabagismo ${ }^{4,5}$.

O estudo, Vigilância de Fatores de Risco e Proteção para Doenças Crônicas por Inquérito Telefônico (Vigitel 2006), mostrou que o número de pessoas com diagnóstico prévio de hipertensão chegou a $23,1 \%$, sendo ligeiramente maior entre as mulheres $(25,5 \%)$ do que entre os homens $(20,3 \%)$. Segundo o estudo, a hipertensão se torna mais comum com a idade, alcançando cerca de $6 \%$ dos indivíduos entre os 18 e 24 anos e cerca de $60 \%$ nas pessoas acima de 65 anos $^{6}$.

Os dados do Vigitel ${ }^{6}$ revelam ainda que a frequência de homens hipertensos variou de 14,8\% (Palmas) e 29,6\% (Rio de Janeiro). O maior percentual foi observado em São Paulo, com 25,1\%, e o menor em São Luís, com 12,5\%. Entre as mulheres, o Rio de Janeiro lidera o número de hipertensas; $34,7 \%$ e por último está a cidade de Palmas, com 14,2\%.

Grande ênfase tem-se dado às medidas não farmacológicas, dentre elas, vem se destacando a prática regular de atividades físicas, componente importante na melhoria da qualidade de vida. Estudos constataram menor morbidade e mortalidade, por doenças do sistema cardiovascular, em indivíduos treinados fisicamente, com benefícios evidentes no subgrupo de hipertensos ${ }^{7}$.

A inatividade física é reconhecidamente um dos mais importantes fatores de risco para as doenças cardiovasculares. $\mathrm{O}$ estilo de vida sedentário, o tabagismo, a hipertensão arterial e a dislipidemia compõem situações passíveis de modificação, para um conjunto de doenças crônico-degenerativas consideradas o principal problema de saúde dos tempos atuais. Dados indicam que 20\% dos adultos são pouco ativos (apenas uma vez por semana) e somente $8 \%$ fazem atividade física regular (três vezes por semana) no Brasil. Isso evidencia a importância que se deve atribuir aos programas de Atividade Física Terapêutica como meio para a abordagem ao hipertenso ${ }^{8}$.

O exercício físico realizado regularmente provoca importantes adaptações autonômicas e hemodinâmicas que vão influenciar o sistema cardiovascular ${ }^{9}$, com o objetivo de manter a homeostasia celular diante do incremento das demandas metabólicas. Há aumento no débito cardíaco, redistribuição no fluxo sanguíneo e elevação da perfusão circulatória para os músculos em atividade ${ }^{10}$.

De acordo com o Conselho Federal de Educação Física - CONFEF a prescrição de atividades físicas necessita da orientação de um Especialista do Exercício - o Profissional de Educação Física é capacitado para prescrever e orientar sessões de atividades físicas com fins educacionais, de treinamento, de prevenção de doenças e promoção da saúde. Nesse sentido, o objetivo deste trabalho foi avaliar e comparar o nível de conhecimento dos Profissionais de Educação Física que atuavam e não atuavam com alunos hipertensos.

\section{PROCEDIMENTOS METODOLÓGICOS}

Estudo descritivo, transversal e quantitativo, realizado no período de outubro de 2005 a abril de 2006, em 84 academias de ginástica em funcionamento e cadastradas no Conselho Regional de Educação Física-CREF-5, em Fortaleza-CE. De acordo com o Conselho Federal de Educação Física - CONFEF, somente as academias cadastradas no CREF podem estar em funcionamento. Este trabalho foi aprovado pelo Comitê de Ética em Pesquisa da Universidade de Fortaleza - UNIFOR ( $\mathrm{n}^{\circ}$ do protocolo 061/2006).

A amostra foi composta por 452 profissionais de Educação Física, de um total de 1.052 inscritos no CREF-5, sendo que 52 recusaram-se a participar do estudo. Sendo assim, o número total foi de 400 , sendo 182 mulheres e 218 homens, independentemente da idade, tempo de formação profissional e que estivessem presentes no momento da visita à academia. Essa amostra corresponde a 38\% do total de profissionais inscritos no CREF-5, até o mês de abril de 2006, da cidade de Fortaleza-CE. Verificamos, também, que na cidade de Fortaleza a maioria dos profissionais que trabalhavam nas academias de ginástica não eram cadastrados no CREF-5.

Inicialmente, realizou-se um levantamento das academias de ginástica cadastradas e o número 
total de profissionais inscritos regularmente no CREF-5. A partir destes dados foram visitadas todas essas academias, sendo solicitada a autorização do coordenador ou do professor responsável pelo estabelecimento. Estas visitas ocorreram nos horários de funcionamento, de forma aleatória. Nas academias em que não foram encontrados os participantes, na primeira visita, os pesquisadores retornaram até duas vezes mais. Os profissionais foram selecionados aleatoriamente, tendo como critério de inclusão ser graduado em Educação Física, estar inscrito regularmente no CREF-5 e trabalhar em academias de ginástica cadastradas no CREF-5.

Para a avaliação do nível de conhecimento do Profissional de Educação Física, frente a alunos com hipertensão arterial nas academias de ginástica, foi elaborado pelos pesquisadores um questionário estruturado com questões abertas e fechadas, direcionadas quanto ao conhecimento de critérios diagnósticos de hipertensão arterial, tipos de exames laboratoriais solicitados, tipo de hipertensão dos alunos, cuidados gerais para a prática de atividade física (solicita avaliação fisica prévia e liberação médica? que situações contra-indicam a atividade física para o aluno hipertenso? verifica a pressão arterial (PA) antes e depois da atividade fisica?) e recomendações para adquirir um melhor controle da hipertensão arterial. As questões abertas foram categorizadas em "corretas" e "incorretas" de acordo com os dados da literatura.

Os questionários foram entregues aos profissionais no momento da visita, sob a supervisão dos pesquisadores que permaneceram no local até que os profissionais respondessem-no por completo. Todos os participantes assinaram um termo de consentimento livre e esclarecido, concordando em participar do estudo.

Os dados foram processados no programa STATA versão 9.0, 2003. Foi realizada uma análise descritiva (frequência, média e desvio padrão da média) e para comparar os dados, foi utilizado o teste Qui-quadrado $\left(\chi^{2}\right)$. O nível de significância adotado foi de $5 \%(\mathrm{p}<0,05)$.

\section{RESULTADOS}

A população estudada foi composta por 400 Profissionais de Educação Física, destes, 302 (75,5\%) atuavam com alunos hipertensos e 98 (24,5\%) não atuavam. As características gerais e formação profissional dos participantes encontram-se descritas na Tabela 1 .
Tabela 1. Características da amostra quanto ao gênero, idade, tempo de formação e especialização.

\begin{tabular}{lc}
\hline Variáveis & $\mathrm{N}(\%)$ \\
\hline Sexo & $182(45,5)$ \\
$\quad$ Feminino & $218(54,5)$ \\
$\quad$ Masculino & $29,5 \pm 4,8$ \\
Idade média dos participantes (anos) & $4,8 \pm 3,9$ \\
Tempo de formação profissional (anos) & \\
Possui especialização (pós-graduação lato & $261(65,3)$ \\
sensu) & $139(34,7)$ \\
$\quad$ Sim & \\
$\quad$ Não & $400(100 \%)$ \\
\hline Total &
\end{tabular}

Quanto ao conhecimento de critérios diagnóstico de hipertensão arterial

Quanto aos critérios de diagnóstico de HA e tipos de exames, observamos que dos 302 profissionais que atuavam, 185 (61,3\%) baseavam-se na informação do aluno e 117 (38,7\%) solicitavam a apresentação de exames comprobatórios do diagnóstico, sendo essa a resposta correta para um melhor diagnóstico da HA.

Comparando-se os tipos de exames solicitados entre os que atuavam e os que não atuavam, constatou-se que não houve diferença significativa entre os participantes. Notamos, na Tabela 2, que aqueles que atuavam com alunos hipertensos não estavam preparados para trabalhar com essa clientela, visto que, a maioria, 37,4\% não sabia informar o tipo de hipertensão dos alunos.

Tabela 2. Conhecimento dos Profissionais de Educação Física quanto aos parâmetros de diagnóstico de hipertensão arterial, tipos de exames solicitados e tipo de hipertensão dos alunos.

\begin{tabular}{lccc}
\hline & $\begin{array}{c}\text { Atuavam com } \\
\text { hipertensos } \\
\mathrm{N}(\%)\end{array}$ & $\begin{array}{c}\text { Não } \\
\text { atuavam } \\
\mathrm{N}(\%)\end{array}$ & $X^{2}$ \\
\hline Parâmetros de diagnóstico de hipertensão arterial & \\
\hline Informação do aluno & $185(61,3)$ & $53(54,1)$ & 0,208 \\
Solicitação de exames & $117(38,7)$ & $45(45,9)$ & \\
\hline Tipos de exames solicitados & $15(12,8)$ & $06(13,3)$ & 0,679 \\
\hline MAPA & $19(16,2)$ & $10(22,2)$ & \\
Eletrocardiograma & $06(5,1)$ & $02(4,4)$ & \\
Ecocardiograma & $12(10,2)$ & $01(2,2)$ & \\
Hemograma & $33(28,2)$ & $11(24,4)$ & \\
Mensuração da PA & $22(18,8)$ & $11(24,4)$ & \\
Teste ergométrico & $10(8,5)$ & $04(8,8)$ & \\
Não respondeu & $100(33,1)$ & & \\
\hline Tipo de Hipertensão dos alunos & & \\
\hline Primária & $89(29,4)$ & & \\
Secundária & $113(37,4)$ & & \\
Não sabe & & & \\
\hline & & & \\
\hline
\end{tabular}


Cuidados gerais para a prática de atividade física Sobre a solicitação de uma avaliação física prévia antes de iniciar um programa de atividade física, 204 $(67,5 \%)$ profissionais que atuavam com hipertensos solicitavam e $98(32,5 \%)$ não. Aqueles que não atuavam, 43 (43,8\%) solicitavam e 55 (56,2\%) não.

Em relação à liberação médica, 198 (65,6\%) solicitavam e 104 (34,4\%) não. Os que não atuavam, $57(58,2 \%)$ solicitavam essa liberação e 41 (41,8\%) não.

A respeito das situações em que os profissionais contra-indicavam a atividade física, 130 (43,1\%) informaram PA elevada, $41(13,6)$ sem acompanhamento médico, $19(6,3)$ treinamento com peso, $47(15,5 \%)$ nenhuma situação e $65(21,5 \%)$ não sabiam informar. Nota-se que não observamos nenhuma diferença estatisticamente significativa entre aqueles que atuavam e não atuavam com hipertensos (Tabela 3).

Tabela 3. Conhecimento dos profissionais quanto às situações que contra-indicavam a atividade física.

\begin{tabular}{lccc}
\hline & $\begin{array}{c}\text { Atuavam } \\
\text { com } \\
\text { hipertensos }\end{array}$ & $\begin{array}{c}\text { Não } \\
\text { atuavam }\end{array}$ & $\mathrm{X}^{2}$ \\
\hline $\begin{array}{l}\text { Situações que } \\
\text { contra-indicavam o } \\
\text { atividade física }\end{array}$ & $\mathrm{N}(\%)$ & $\mathrm{N}(\%)$ & \\
$\begin{array}{l}\text { PA elevada } \\
\begin{array}{l}\text { Paciente sem acom- } \\
\text { panhamento médico }\end{array}\end{array}$ & $41(13,6)$ & $13(13,2)$ & \\
$\begin{array}{l}\text { Treinamento com } \\
\text { peso }\end{array}$ & $19(6,3)$ & $7(7,1)$ & \\
Nenhuma situação & $47(15,5)$ & $20(20,4)$ & \\
Não sabe & $65(21,5)$ & $28(28,5)$ & \\
\hline
\end{tabular}

Em relação à mensuração da $\mathrm{PA}$ antes e depois da atividade física, observamos que $62,3 \%$ dos profissionais que atuavam com hipertensos não verificava a PA antes e depois da atividade física. Quando questionados sobre o motivo pelo qual não verificavam a PA, $56(29,7)$ afirmaram que a academia não fornecia aparelho de pressão e 40 $(21,3)$ não sabiam verificar a PA (Tabela 4).

Quando questionados sobre as principais recomendações para adquirir um melhor controle da hipertensão, verificamos que não houve diferença significativa entre aqueles que atuavam e não atuavam com hipertensos (Tabela 5).

\section{DISCUSSÃO}

As alterações no perfil de morbidade que a sociedade vem sofrendo, as alterações nos padrões de
Tabela 4. Mensuração da PA antes e depois da atividade física e motivo pelo qual os Profissionais de Educação Física que atuavam com hipertensos não verificavam.

\begin{tabular}{lc}
\hline & $\begin{array}{c}\text { Atuavam com } \\
\text { hipertensos } \\
\mathrm{N}(\%)\end{array}$ \\
\hline $\begin{array}{l}\text { Verifica a PA antes e depois da } \\
\text { atividade física }\end{array}$ & $114(37,7)$ \\
Sim & $188(62,3)$ \\
Não & \\
Motivo pelo qual não verificam a PA & $56(29,7)$ \\
$\begin{array}{l}\text { Academia não fornece aparelho de } \\
\text { pressão }\end{array}$ & $28(14,8)$ \\
Falta tempo & $30(15,9)$ \\
O aluno já é acompanhado pelo & \\
médico & $34(18,3)$ \\
Não acham necessário & $40(21,3)$ \\
\hline Não sabem
\end{tabular}

Tabela 5. Recomendações para adquirir um melhor controle da hipertensão arterial.

\begin{tabular}{lccc}
\hline & $\begin{array}{c}\text { Atuavam com } \\
\text { hipertensos } \\
\mathrm{N}(\%)\end{array}$ & $\begin{array}{c}\text { Não } \\
\text { atuavam } \\
\mathrm{N}(\%)\end{array}$ & $\mathrm{X}^{2}$ \\
\hline $\begin{array}{l}\text { Recomendações } \\
\text { Consultar o médico } \\
\text { regularmente }\end{array}$ & $32(10,60)$ & $06(6,12)$ & 0,020 \\
$\begin{array}{l}\text { Controlar a PA e a } \\
\text { alimentação }\end{array}$ & $60(19,87)$ & $12(12,24)$ \\
$\begin{array}{l}\text { Controlar somente a } \\
\text { alimentação }\end{array}$ & $36(11,92)$ & $09(9,18)$ \\
$\begin{array}{l}\text { Realizar atividade } \\
\text { física }\end{array}$ & $93(30,79)$ & $30(30,61)$ \\
$\begin{array}{l}\text { Tomar a medicação } \\
\text { corretamente }\end{array}$ & $23(7,62)$ & $06(6,12)$ \\
\begin{tabular}{l} 
Não sabe \\
\hline
\end{tabular} & $58(19,21)$ & $35(35,71)$ \\
\hline
\end{tabular}

ingestão alimentar, inatividade física e aumento das doenças crônicas, entre elas a hipertensão arterial e o diabetes mellitus, fazem com que o profissional de Educação Física se depare com novos grupos de praticantes de exercícios fisicos, com necessidades e cuidados especiais. Isto requer que o profissional de Educação Fisica esteja cada vez mais capacitado para atender de maneira segura esta população.

Os resultados aqui apresentados mostraram que a maioria dos profissionais que atuava com hipertensos apresentou uma carência de conhecimento sobre HA. Sabemos que o ambiente de trabalho é um fator estimulador para um melhor desempenho profissional. Os coordenadores das academias poderiam estimular os profissionais de Educação Fisica através de cursos de aprimoramento e palestras sobre a temática. Notamos, ainda, que as condições de trabalho de algumas academias eram precárias, 
sem aparelhos adequados, até mesmo com falta de aparelho para verificar a PA e sem uma assistência básica de primeiros socorros.

O questionário utilizado não foi submetido a um processo formal de validação, o que pode ser considerado uma limitação do presente estudo. Por outro lado, as perguntas foram facilmente compreendidas pelos profissionais e não foram detectadas inconsistências marcantes nas respostas. Outro aspecto foi a recusa de 52 profissionais em participar do estudo e o fato de muitas academias não estarem cadastradas no CREF-5.

Quanto ao diagnóstico de HA, os exames mais solicitados foram a mensuração da PA e teste ergométrico. Segundo as IV Diretrizes de Hipertensão Arterial ${ }^{11}$, a medida da PA é o elemento-chave para o estabelecimento do diagnóstico da HA e a avaliação da eficácia do tratamento.

Um estudo realizado em Pelotas observou que as principais provas para o diagnóstico de HA estavam constituídas da mensuração da PA, exame de urina, creatinina e potássio sérico, colesterol total, glicemia em jejum e eletrocardiograma de repouso ${ }^{12}$.

Antes de iniciarem programas regulares de exercício físico, os hipertensos devem ser submetidos à avaliação clínica especializada, exame préparticipação e recomendações médicas relacionadas aos exercícios. Hipertensos em estágio 3 só devem iniciar o exercício após controle da $\mathrm{PA}^{13-15}$.

A Sociedade Brasileira de Cardiologia recomenda que os indivíduos hipertensos iniciem programas de exercício físico, desde que submetidos à avaliação clínica prévia. Os exercícios devem ser de intensidade moderada, de três a seis vezes por semana, em sessões de 30 a 60 minutos de duração, realizados com frequência cardíaca entre $60 \%$ e $80 \%$ da máxima ou entre $50 \%$ e $70 \%$ do consumo máximo de oxigênio ${ }^{16}$.

No estudo com o objetivo de analisar o conhecimento dos profissionais de Educação Física em relação à atividade física como promotora da saúde, observou-se que eles obtiveram 75\% de êxito em relação ao conhecimento sobre a prescrição de exercícios para a saúde, demonstrando que os mesmos apresentam conhecimento ao prescrever exercício. Já em relação aos conhecimentos sobre os fatores de riscos, eles apresentaram $62,5 \%$ de acertos ${ }^{17}$.

Antes de iniciar um programa de exercício, o indivíduo com HA deve ser submetido a uma avaliação médica detalhada, com estudos diagnósticos apropriados, e somente após a liberação do médico, estará apto a realizar qualquer atividade física ${ }^{18}$.

Ferreira, Matsudo e Braggion ${ }^{19}$ ressaltaram a importância da orientação médica sobre a prática de atividades físicas como forma não só da prevenção primária das doenças crônicas, mas também da prevenção secundária em pacientes com doenças cardiovasculares e outras enfermidades.

Estudos randomizados mostraram efeitos indesejáveis do tratamento farmacológico em subgrupos de pacientes com HA, sugerindo uma mudança na abordagem do tratamento dos mesmos. $\mathrm{O}$ efeito do exercício físico sobre os níveis de repouso da pressão arterial de grau leve a moderado é especialmente importante, uma vez que o paciente hipertenso pode diminuir a dosagem dos seus medicamentos antihipertensivos ou até ter a sua pressão arterial controlada, sem a adoção de medidas farmacológicas ${ }^{20,21}$

Estudo envolvendo 217 pacientes de ambos os sexos, com idade variando de 35 a 83 anos, mostrou que a adesão a medidas não farmacológicas, dentre as quais a prática de exercício físico, promoveu sensível efeito na redução dos níveis pressóricos ${ }^{22}$

A prescrição do exercício para indivíduos hipertensos é, em geral, semelhante ao que se recomenda para desenvolver e manter a aptidão cardiorrespiratória de adultos normotensos ${ }^{23}$.

Em um estudo foram avaliados 27 indivíduos, $88,9 \%$ demonstraram ter conhecimentos sobre a finalidade da prescrição de exercícios físicos para a saúde, e apenas $11,1 \%$ demonstraram a falta desse conhecimento ${ }^{17}$.

As variáveis cardiovasculares vêm sendo monitoradas para indicar diretamente o impacto da intensidade de esforço e da modalidade de exercício sobre a taxa de trabalho do miocárdio ${ }^{24}$. A frequência cardíaca (FC) e PA são as variáveis mais utilizadas, e associadas, fornecem o Duplo produto (DP) que permite avaliar de forma indireta o trabalho do miocárdio ${ }^{25}$.

Observamos nesse estudo que alguns profissionais contra-indicavam o treinamento resistido para hipertensos. Existe na literatura um grande número de estudos relacionados às respostas cardiovasculares agudas durante exercícios físicos. Eles demonstram que os exercícios resistidos (ER) apresentam menor Duplo produto (DP) do que exercícios aeróbicos ${ }^{26}$. Farinatti e Assis ${ }^{26}$ avaliaram 18 indivíduos em exercícios contra resistência em um, seis e 20 repetições máximas (RM) e um treinamento aeróbico contínuo no cicloergômetro durante 20 minutos a $75-80 \%$ da FC de reserva. Nos ER o maior DP foi registrado em 20RM, seguido de 6RM e finalmente, o de 1RM, registrando o menor valor para o DP. Sendo assim, nos ER o aumento do DP ficou mais associado ao número de repetições do que à carga 
e no trabalho aeróbio, foi registrado um maior DP quando comparado aos ER. Concluiu-se que os ER, independente da intensidade, impõem uma menor solicitação cardíaca que a atividade aeróbica.

A prática de atividade física regular é uma estratégia eficaz para controlar os níveis pressóricos. Os dados da literatura ressaltam a importância dos benefícios para a saúde que podem ser conseguidos com uma alimentação saudável e atividade física ${ }^{27}$.

Paffenbarger et al. ${ }^{28}$, em um seguimento de 6 a 10 anos, de 15.000 indivíduos de Harvard, constataram que os que praticavam exercício físico de forma regular apresentavam risco 35\% menor de desenvolver hipertensão arterial do que os indivíduos sedentários.

Em outro estudo, Paffenbarger et al. ${ }^{29}$ seguiram 10.260 diplomados de Harvard, por mais de uma década e constataram uma relação inversa entre a prática de atividade física e o risco de morte por todas as causas. Ao comparar os que iniciaram atividade esportiva moderadamente vigorosa, com aqueles que não participaram desse tipo de treinamento, observaram redução de $23 \%$ no risco de morte.

Verenguer ${ }^{30}$,quando discute o mundo do trabalho e as relações de trabalho estabelecidas pelos profissionais de Educação Física, aponta para a carência de estudos, afirmando que essa temática é atual e pertinente.

\section{CONCLUSÃO}

Evidencia-se a necessidade de se implementar um programa de capacitação para esses profissionais, pois foi observado que os participantes do presente estudo não apresentaram um conhecimento satisfatório em algumas questões como: tipos de exames para o diagnóstico da HA, tipos de hipertensão dos seus alunos, situações que contra-indicavam a atividade física e mensuração da PA antes e depois da atividade fisica.

Existem vários fatores que poderiam contribuir para uma melhor qualificação desses profissionais, como por exemplo, a realização de cursos de aprimoramento e de atualização oferecidos pelo Conselho Regional de Educação Física, a busca do profissional pelo conhecimento, participando de cursos, congressos e pesquisas sobre HA e mudanças das diretrizes curriculares para melhorar a formação do futuro profissional que irá trabalhar na área de prevenção e tratamento de várias doenças com a prescrição de exercícios físicos.

Sendo assim, o primeiro direcionamento para um melhor conhecimento deveria começar nos cursos de bacharelado com uma atenção especial para disciplinas da área do treinamento físico para grupos em situações diferenciadas, como os hipertensos, por exemplo.

Espera-se que mudanças relacionadas à formação acadêmica e atuação profissional na área se concretizem, especialmente, no que se refere à identidade de cada habilitação do curso e sua articulação com os campos de atuação, contribuindo para a valorização profissional e social.

\section{REFERENCIAS BIBLIOGRÁFICAS}

1. Chobanian AV, Bakris GL, Black HR, Cusham WC, Green LA, Izzo JL Jr, et al. National High Blood Pressure Education Program Coordinating Committee. The seventh report of the Joint National Committee on Prevention, Detection, Evaluation, and Treatment of High Blood Pressure: the JNC 7 report. JAMA 2003;289(19):2560-2572.

2. Fagard RH. Exercise characteristics and the blood pressure response to dynamic physical training. Med Sci Sports Exerc 2001;33(6):484-492.

3. American College of Sports Medicine. Diretrizes do ACSM para os testes de esforço e sua prescrição. $6^{a}$ ed. Rio de Janeiro: Guanabara Koogan; 2000.

4. Jardim PCBV, Gondim MRP, Monego ET, Moreira HG, Vitorino PVO, Souza WKSB, et al. Hipertensão arterial e alguns fatores de risco em uma capital brasileira. Arq Bras Cardiol 2007;88(4):452-457.

5. Brasil MS. Coordenação de Doenças Cardiovasculares. Controle de hipertensão arterial: uma proposta de integração ensino-serviço. Rio de Janeiro: CDCV/ NUTES; 1993.

6. Brasil Ministério da Saúde. Secretaria de Vigilância em Saúde, Secretaria de Gestão Estratégica e Participativa. Vigitel Brasil 2006: Vigilância de Fatores de Risco e Proteção para Doenças Crônicas por Inquérito Telefônico. Brasília, 2007

7. Lewington S, Clarke R, Qizilbash N, Peto R, Collins R. Agespecific relevance of usual blood pressure to vascular mortality. Lancet 2002;360(9349):1903-1913.

8. Godoy M. I Consenso Nacional de Reabilitação Cardiovascular. Arq Bras Cardiol 1997;69(4):267-291.

9. Staessen JA, Thijs L, Fagard R, O'Brien ET, Clement DL, Leeuw PW, et al. Predicting cardiovascular risk using conventional vs. ambulatory blood pressure in older patients with systolic hypertension. JAMA 1999; 282(11):539-546.

10. Rondon MUPB, Brum PC. Exercício físico como tratamento não-farmacológico da hipertensão arterial. Rev Bras Hipertens 2003;10(2):134-139.

11. Francisco AG, Jardim PCBV, Brasil MAA, Souza ALL, Freitas HF, Taniguchi LM, et al . Prevalência de transtorno depressivo maior em centro de referência no tratamento de hipertensão arterial. Rev Psiquiatr Rio Gd Sul 2007;29(2):161-168.

12. Sturmer G, Soares JD, Olinto MTA, Menezes AMB, Gigante DP, Macedo S. O manejo não medicamentoso 
da hipertensão arterial sistêmica no Sul do Brasil. Cad Saúde Pública 2006;22(8):1727-1737.

13. Whelton SP, Chin A, Xin X, He J. Effects of aerobic exercise on blood pressure: A meta-analysis of randomized controlled trials. Ann/intern Med 2002;136(7):493-503.

14. Cornelissen VA, Fagard RH. Effect of resistance training on resting blood pressure: a meta-analysis of randomized controlled trials. J Hypertens 2005;23(2):251-259.

15. Myers J, Prakash M, Froelicher V, Do D, Partington $\mathrm{S}$, Atwood JE. Exercise capacity and mortality among men referred for exercise testing. N Engl J Med 2002;346(11):793-801.

16. Sociedade Brasileira de Cardiologia. IV Diretrizes Brasileiras de Hipertensão. Arq Bras Cardiol 2004; 82 (suppl): 13-14.

17. Cabral IA, Sousa MAM, Raydan FPS. Análise do conhecimento dos profissionais de educação física em relação à atividade física como promotora da saúde. Movimentum - Revista Digital de Educação Física 2007;2(2):1-11.

18. Campos EP, Leite SC. Fatores terapêuticos de um programa continuado e integrado de atendimento ao hipertenso. Rev Prática Medica 1990;101(9):9-14.

19. Ferreira M, Matsudo S, Matsudo V, Braggion G. Efeitos de um programa de orientação de atividade física e nutricional sobre o nível de atividade física de mulheres fisicamente ativas de 50 a 72 anos de idade. Rev Bras Med Esporte 2005;11(3):172-176.

20. Gusmão JL, Ginani GF, Silva GV, Ortega KC, Mion $\mathrm{JrD}$. Adesão ao tratamento em hipertensão arterial sistólica isolada. Rev Bras Hipertens 2009;16(1):38-43.

21. Monteiro MF, Filho DCS. Exercício físico e o controle da pressão arterial. Rev Bras Med Esporte 2004;10(6):513-516.

22. Ferreira KVS, Melo AMCA, Sobral Filho DC, Arruda IKG, Diniz AS, Toscano CHH. Impacto das Modificações no Estilo de Vida no Controle da Hipertensão. Arq Bras Cardiol 1999;73(4):110-111.

23. Pinto VLM, Meirelles LR, Farinatti PTV. Influência de programas não-formais de exercícios (doméstico e comunitário) sobre a aptidão física, pressão arterial e variáveis bioquímicas em pacientes hipertensos. Rev Bras Med Esporte 2003; 9(3):267-274
24. Polito MD, Farinatti PTV, Lira VA, Nóbrega ACL. Blood pressure assessment during resistance exercise: comparison between auscultation and Finapres. Blood Press Monit 2007;12(2):81-86.

25. Leite TC, Farinatti PTV. Estudo da freqüência cardíaca, pressão arterial e duplo-produto em exercícios resistidos diversos para grupamentos musculares semelhantes. Rev Bras Fis Exerc 2003;2(1):29-49.

26. Farinatti PTV, Assis BFCB. Estudo da freqüência cardíaca, pressão arterial e duplo-produto em exercícios contra-resistência e aeróbico contínuo. Rev Bras Ativ Física Saúde 2000;5(2):5-16.

27. Brasil. Ministério da Saúde. Physical activity and life quality contribution in order to obtain a better healthly lifestyle. Rev Saúde Pública 2002;36(2):254-256.

28. Paffenbarger RS, Wing AL, Hyde RT. Physical exercise and incidence of hypertension in college alumni. Am J Epidemiol 1983;117(6):245-57.

29. Paffenbarger RSJr, Hyde RT, Wing AL, Lee IM, Jung DL, Kampert JE. The association of changes in physical-activity level and other lifestyle characteristics with mortality among men. N Engl J Med 1993;328(6):538-545.

30. Verenguer RCG. Mercado de trabalho em educação física: reestruturação produtiva, relações de trabalho e intervenção profissional. Rev Mackenzie Educ Fís Esporte 2005;4(4):39-54.

\section{Endereço para correspondência}

Luciana Zaranza Monteiro

Rua Rui Barbosa 740 apt. 237

14015-120, Ribeirão Preto, SP. Brasil.

E-mail: lucianazaranza@usp.br 